\title{
Analytical Solutions to the Steady State Poisson-Nernst-Planck Equations in Electrobiochemical Systems
}

\author{
A. Samin ${ }^{1} \&$ V. V. Subramaniam ${ }^{1}$ \\ ${ }^{1}$ Applied Physics Laboratory, Department of Mechanical \& Aerospace Engineering, The Ohio State University, \\ Columbus, Ohio 43210, USA \\ Correspondence: A. Samin, Applied Physics Laboratory, Department of Mechanical \& Aerospace Engineering, \\ The Ohio State University, Columbus, Ohio 43210, USA. E-mail: samin.2@ buckeyemail.osu.edu
}

\author{
Received: January 30, 2015 Accepted: February 10, 2015 Online Published: February 11, 2015 \\ doi:10.5539/apr.v7n2p40 URL: http://dx.doi.org/10.5539/apr.v7n2p40
}

\begin{abstract}
The Poisson-Nernst-Planck equations are relevant in numerous electrobiochemical applications. In this paper, we provide analytical solutions to the steady state Poisson-Nernst-Planck (PNP) systems of equations for situations relevant to applications involving bioelectric dressings and bandages. The PNP system of equations is analyzed for two ionic species (one positively charged and the other negatively charged) both in the one-dimensional and two dimensional cases. The equations were formulated, non-dimensionalized, and an order of magnitude analysis was performed. Additionally, the method of singular perturbations was utilized in the two dimensional case. In the one-dimensional case, an exact solution is obtained while in the two-dimensional case an asymptotic solution is obtained. Both analytical solutions are compared with numerical solutions of the equations, and exhibit good agreement. The analytical solutions for the benchmark problems presented here are useful for verifying numerical solutions to more complex problems, and may also enable simple interpretation of experimental data for electrobiochemical systems.
\end{abstract}

\section{Introduction}

In many electrochemical and electrobiochemical applications, the continuum governing equations describing the concentrations of ionic species and their transport under the action of electric fields are the Poisson-Nernst-Planck (PNP) Equations. Despite the fact that these equations have been well known and studied for over a century, they remain of current interest because of applications ranging from batteries (Torabi \& Aliakbar, 2012; Venkatraman \& Van Zee, 2007), bioelectric dressings used in wound healing (Banerjee et al., 2014), diffusion of charged species through ion channels in cell membranes and ion selective membranes (Coalson \& Kurnikova, 2005; Fíla \& Bouzek, 2003), electro-osmosis in micro and nano-channel systems (Hrdlička, Červenka, Přibyl, \& Šnita, 2010), transport of charge carriers in semiconductors (Markowich, 1986), ionic current rectification through charged micro and nano channels (Chein \& Chung, 2013) to as diverse a field as deterioration of reinforced concrete structures due to diffusion and attack by the chloride ion (K. Krabbenhøft, \& J. Krabbenhøft, 2008). In their most general form (unsteady, 3-D), the PNP equations are usually solved numerically. Nevertheless, analytical and approximate closed form solutions to benchmark problems can be useful in validating numerical solutions, and in enabling a fundamental understanding of the influence of experimental parameters on ionic diffusion.

Exact analytical solutions to the PNP equations have been obtained for various situations. In addition, approximate solutions using singular perturbation analyses and numerical solutions have been also been reported. These analytical and approximate solutions can be broadly categorized as considering (1) 1-D, steady-state, two species (Barcilon, Chen, Eisenberg, \& Jerome, 1997; Golovnev \& Trimper, 2010; Liu, 2005; Singer, Gillespie, Norbury, \& Eisenberg, 2008), (2) 1-D, unsteady, two species (Golovnev \& Trimper, 2011), (3) 1-D, unsteady, single species (Schönke, 2012), and (4) 2-D and 3-D, unsteady, single species cases (Schönke, 2012). Moreover, the analytical solutions obtained for these different cases vary in the problem formulation as far as boundary conditions, scaling, and assumptions are concerned. For instance, elegant closed form solutions have been obtained in the case of a single ionic species for the unsteady 1-D, 2-D, and 3-D PNP equations (Schönke, 2012). For the 1-D, unsteady case with a single ionic species, the PNP equations have been shown to reduce to the scalar Burger's equation (Schönke, 2012). However, the single species PNP equations lead to incorrect values 
for the diffusivity inferred from migration experiments because of neglect of the need for quasi-neutrality (K. Krabbenhøft, \& J. Krabbenhøft, 2008). In this paper, we consider a benchmark problem pertaining to electrobiochemical systems, specifically bioelectric dressings used in wound healing (Banerjee et al., 2014), which involve the two-species, steady-state PNP equations in 1-D and 2D. Approximate solutions to these equations are obtained using order of magnitude analysis and the singular perturbation method for the 2-D case.

This paper is organized as follows. The following section discusses formulation of a model 1-D, steady-state benchmark problem with two ionic species and its analytical solution. The two-species PNP equations for a model two dimensional problem, associated scaling, and their approximate solution using the method of singular perturbations are given in Section 3. A summary along with the conclusions are given in Section 4 .

\section{Poisson-Nernst-Planck Equations in One-Dimension}

The PNP system for two ionic species (one a singly charged positive ion and the other a singly charged negative ion) in a linear, isotropic, homogeneous medium is given by:

$$
\begin{gathered}
\frac{\partial p^{*}}{\partial t^{*}}=D_{p} \frac{\partial^{2} p^{*}}{\partial x^{* 2}}+\mu_{p} \frac{\partial}{\partial x^{*}}\left(p^{*} \frac{\partial \varphi^{*}}{\partial x^{*}}\right) \\
\frac{\partial n^{*}}{\partial t^{*}}=D_{n} \frac{\partial^{2} n^{*}}{\partial x^{* 2}}+\mu_{p} \frac{\partial}{\partial x^{*}}\left(n^{*} \frac{\partial \varphi^{*}}{\partial x^{*}}\right) \\
\frac{\partial^{2} \varphi^{*}}{\partial x^{* 2}}=-\frac{e}{\varepsilon}\left(p^{*}-n^{*}\right)
\end{gathered}
$$

where the * denotes a dimensional quantity, $\mathrm{p}$ and $\mathrm{n}$ are the number densities of positive and negative ions, $\mathrm{D}_{\mathrm{p}}$ and $D_{n}$ are the respective ion diffusivities, $\mu_{p}$ and $\mu_{n}$ are the respective ion mobilities, $\varphi$ is the electric potential, e is the fundamental charge of an electron, $\mathrm{k}$ is Boltzmann's constant, $\varepsilon$ is the permittivity of the medium containing the ions, and $\mathrm{T}$ is the temperature (taken to be a constant here).

\subsection{Model 1-D Problem}

\subsubsection{Formulation of Problem and Scaling}

Consider the model problem of a linear, isotropic, homogeneous, isothermal medium in which are present positive ions of number density $\mathrm{p}$ and negative ions of number density $\mathrm{n}$. Suppose there are two electrodes immersed in the medium which are not connected to an external circuit. Such a situation can arise for example under open circuit conditions in a battery (Torabi \& Aliakbar, 2012; Venkatraman \& Van Zee, 2007), or in a 1-D analog of a bioelectric dressing in contact with water or wound exudate (Banerjee et al., 2014). Furthermore, suppose that there is a constant generation of species $\mathrm{n}$ at one of the electrodes, such as the generation of $\mathrm{OH}^{-}$ ions at the oxide surface of a silver oxide electrode (Torabi \& Aliakbar, 2012). The governing equations (1-3) apply to this case, re-written by relating the mobilities to the ion diffusivities via the Einstein relation $\mu=e Z D / k T$, where $Z=+1$ for the positive ions and $Z=-1$ for the negative ions:

$$
\begin{gathered}
\frac{\partial p^{*}}{\partial t^{*}}=D_{p} \frac{\partial^{2} p^{*}}{\partial x^{* 2}}+\frac{e D_{p}}{k T} \frac{\partial}{\partial x^{*}}\left(p^{*} \frac{\partial \varphi^{*}}{\partial x^{*}}\right) \\
\frac{\partial n^{*}}{\partial t^{*}}=D_{n} \frac{\partial^{2} n^{*}}{\partial x^{* 2}}+\frac{e D_{p}}{k T} \frac{\partial}{\partial x^{*}}\left(n^{*} \frac{\partial \varphi^{*}}{\partial x^{*}}\right) \\
\frac{\partial^{2} \varphi^{*}}{\partial x^{* 2}}=-\frac{e}{\varepsilon}\left(p^{*}-n^{*}\right)
\end{gathered}
$$

Introducing a reference length scale $\mathrm{L}_{\text {ref }}$, reference time scale $\mathrm{t}_{\text {ref }}$, reference number density $\mathrm{n}_{\text {ref }}$, and reference potential $\varphi_{\text {ref }}$, these equations can be non-dimensionalized to yield:

$$
\begin{gathered}
\Pi_{1} \frac{\partial p}{\partial t}=\frac{\partial^{2} p}{\partial x^{2}}+\Pi_{2} \frac{\partial}{\partial x}\left(p \frac{\partial \varphi}{\partial x}\right) \\
\Pi_{3} \frac{\partial n}{\partial t}=\frac{\partial^{2} n}{\partial x^{2}}+\Pi_{2} \frac{\partial}{\partial x}\left(n \frac{\partial \varphi}{\partial x}\right) \\
\frac{\partial^{2} \varphi}{\partial x^{2}}=-\Pi_{4}(p-n)
\end{gathered}
$$


where $\Pi_{1}=L_{r e f}^{2} / t_{r e f} D_{p}, \Pi_{2}=e \varphi_{r e f} / k T, \Pi_{3}=L_{r e f}^{2} / t_{r e f} D_{n}$, and $\Pi_{4} e n_{r e f} L_{r e f}^{2} / \varepsilon \varphi_{r e f}$. For the case of a bioelectric dressing, $\mathrm{L}_{\mathrm{ref}} \sim 1 \mathrm{~mm}, \mathrm{D}_{\mathrm{OH}^{-}} \sim 4.56 \times 10^{-3} \mathrm{~mm}^{2} / \mathrm{s}$ (Lee \& Rasaiah, 2011), and $\mathrm{t}_{\mathrm{ref}} \gg 10^{3} \mathrm{~s}$ so that $\Pi_{1} \ll 1$ as is $\Pi_{3}$. Moreover, $\varphi_{\text {ref }} \sim 1 \mathrm{~V}$ and $\mathrm{T} \sim 300 \mathrm{~K}$ so that $\Pi_{2} \sim \mathrm{O}(10)$. Therefore, for the problems of interest, the PNP equations reduce to their steady state form:

$$
\begin{gathered}
\frac{\partial^{2} p}{\partial x^{2}}+\Pi_{2} \frac{\partial}{\partial x}\left(p \frac{\partial \varphi}{\partial x}\right)=0 \\
\frac{\partial^{2} n}{\partial x^{2}}+\Pi_{2} \frac{\partial}{\partial x}\left(n \frac{\partial \varphi}{\partial x}\right)=0 \\
\frac{\partial^{2} \varphi}{\partial x^{2}}=-\Pi_{4}(p-n)
\end{gathered}
$$

Equations (10) and (11) may be added and subtracted separately, yielding:

$$
\begin{gathered}
\frac{\partial^{2} P}{\partial x^{2}}+\Pi_{2} \frac{\partial}{\partial x}\left(N \frac{\partial \varphi}{\partial x}\right)=0 \\
\frac{\partial^{2} N}{\partial x^{2}}+\Pi_{2} \frac{\partial}{\partial x}\left(P \frac{\partial \varphi}{\partial x}\right)=0 \\
\frac{\partial^{2} \varphi}{\partial x^{2}}=-\Pi_{4} N
\end{gathered}
$$

where $\mathrm{P}=\mathrm{p}+\mathrm{n}$ and $\mathrm{N}=\mathrm{p}-\mathrm{n}$. Note that if quasi-neutrality prevails, $\mathrm{N}=0$, i.e. $\mathrm{p}=\mathrm{n}$, resulting in the familiar ambipolar diffusion equation for species concentration with zero electric field and with equations (13-15) identically satisfied.

There are two possible choices for $\phi_{\text {ref. }}$. If we set $\varphi_{\text {ref }}=\mathrm{kT} / \mathrm{e}$, then $\Pi_{2}=1$ and $\Pi_{4}=L_{r e f}^{2} / \lambda_{D}^{2}$, where $\lambda_{D}=\left(\varepsilon k T / e^{2} n_{r e f}\right)^{1 / 2}$ is the Debye length. It should be mentioned here that the appearance of the Debye length in this analysis agrees with previous studies that also naturally led to the rise of the Debye length quantity in non-dimensionalization (Hrdlička, Červenka, Přibyl, \& Šnita, 2010). This case has already been considered in the literature for ion channels in membranes and an approximate solution using singular perturbation methods has been obtained (Liu, 2005; Singer, Gillespie, Norbury, \& Eisenberg, 2008). In the present case of a bioelectric dressing however, $\varphi_{\text {ref }}$ is taken to be specified, e.g. the open circuit potential difference across the silver oxide and zinc dots in the bioelectric dressing is on the order of $0.1 \mathrm{~V}$ (Banerjee et al., 2014), and $\mathrm{L}_{\text {ref }}$ is the spacing between the electrodes which is on the order of millimeters in the case of the bioelectric dressing. Thus, $\Pi_{2}=e \varphi_{\text {ref }} / k T \sim 4$ for $\varphi_{\text {ref }}=0.1 \mathrm{~V}$ and $\mathrm{T}=300 \mathrm{~K}$, and $\Pi_{4}=e n_{\text {ref }} L_{r e f}^{2} / \varepsilon \varphi_{r e f} \sim 10^{6}$ for $\mathrm{L}_{\mathrm{ref}}=1 \mathrm{~mm}, \varepsilon \sim$ $80 \times 10^{-12} \mathrm{~F} / \mathrm{m}$, and $\mathrm{n}_{\mathrm{ref}} \sim 10^{20} \mathrm{~m}^{-3}$. The latter values for $\varepsilon$ and $\mathrm{n}_{\text {ref }}$ are based on the medium being physiological saline ( $\mathrm{pH}$ 7.4) from which we obtain $\mathrm{n}_{\mathrm{OH}}{ }^{-} \sim 10^{20} \mathrm{~m}^{-3}$ and $\mathrm{n}_{\mathrm{H}}{ }^{+} \sim 10^{19} \mathrm{~m}^{-3}$. The solution for this case is given next.

\subsubsection{Solution of the 1-D Steady State PNP Equations}

For the conditions where the open circuit voltage and distance between electrodes are specified as in the case of a bioelectric dressing [3], $\Pi_{2}=e \varphi_{\text {ref }} / k T \sim 4$ and $\Pi_{4}=e n_{\text {ref }} L_{r e f}^{2} / \varepsilon \varphi_{\text {ref }} \sim 10^{6}$. The governing equations (13)-(15) may then be re-written as:

$$
\begin{gathered}
\mu \frac{\partial^{2} P}{\partial x^{2}}+\frac{\partial}{\partial x}\left(N \frac{\partial \varphi}{\partial x}\right)=0 \\
\mu \frac{\partial^{2} N}{\partial x^{2}}+\frac{\partial}{\partial x}\left(P \frac{\partial \varphi}{\partial x}\right)=0 \\
\lambda \frac{\partial^{2} \varphi}{\partial x^{2}}=-N
\end{gathered}
$$

where $\mu=1 / \Pi_{2} \sim \mathrm{O}\left(10^{-1}\right)$ and $\lambda=1 / \Pi_{4} \sim \mathrm{O}\left(10^{-6}\right)$. The boundary conditions are taken to be $\mathrm{P}(0)=\mathrm{P}_{\mathrm{L}}, \mathrm{P}(1)=\mathrm{P}_{\mathrm{R}}$, $\mathrm{N}(0)=\mathrm{N}_{\mathrm{L}}, \mathrm{N}(1)=\mathrm{N}_{\mathrm{R}}, \varphi(0)=\varphi_{\mathrm{L}}$, and $\varphi(1)=\varphi_{\mathrm{R}}$. Since, $\lambda$ is on the order of $\mathrm{O}\left(10^{-6}\right)$, then from equation (18) we may conclude that $N \approx 0$. This analysis then yields from equation (16) and from using the boundary conditions, a simple expression for $\mathrm{P}(\mathrm{x})$ :

$$
P(x)=(P(1)-P(0)) x+P(0)
$$


Using this result in equation (17) and applying the boundary conditions for the electric potential, the solution for the non-dimensionalized electric potential is:

$$
\varphi(x)=\frac{C}{(P(1)-P(0))} \ln ((P(1)-P(0)) x+P(0))+D
$$

where

$$
\begin{gathered}
C=\frac{(P(1)-P(0))(\varphi(1)-\varphi(0))}{\ln (P(0))-\ln (P(1))} \\
D=\varphi(1)-\frac{C}{(P(1)-P(0))} \ln (P(1))
\end{gathered}
$$

Such a logarithmic dependence of the electric potential on $\mathrm{x}$ is consistent with previous studies on the one-dimensional PNP system (Singer, Gillespie, Norbury, \& Eisenberg, 2008). Figures 1 through 3 show the numerical solutions which result from solving the full PNP system (Equations 16-18) for the boundary conditions of this model problem $(\mathrm{P}(0)=0.2, \mathrm{P}(1)=0.8, \varphi(0)=0.4, \varphi(1)=1.4, \mathrm{~N}(0)=-0.005$, and $\mathrm{N}(1)=0)$, as well as the analytical solutions given by equations (18)-(20). It can be seen that good agreement is found between the analytical and numerical solutions.

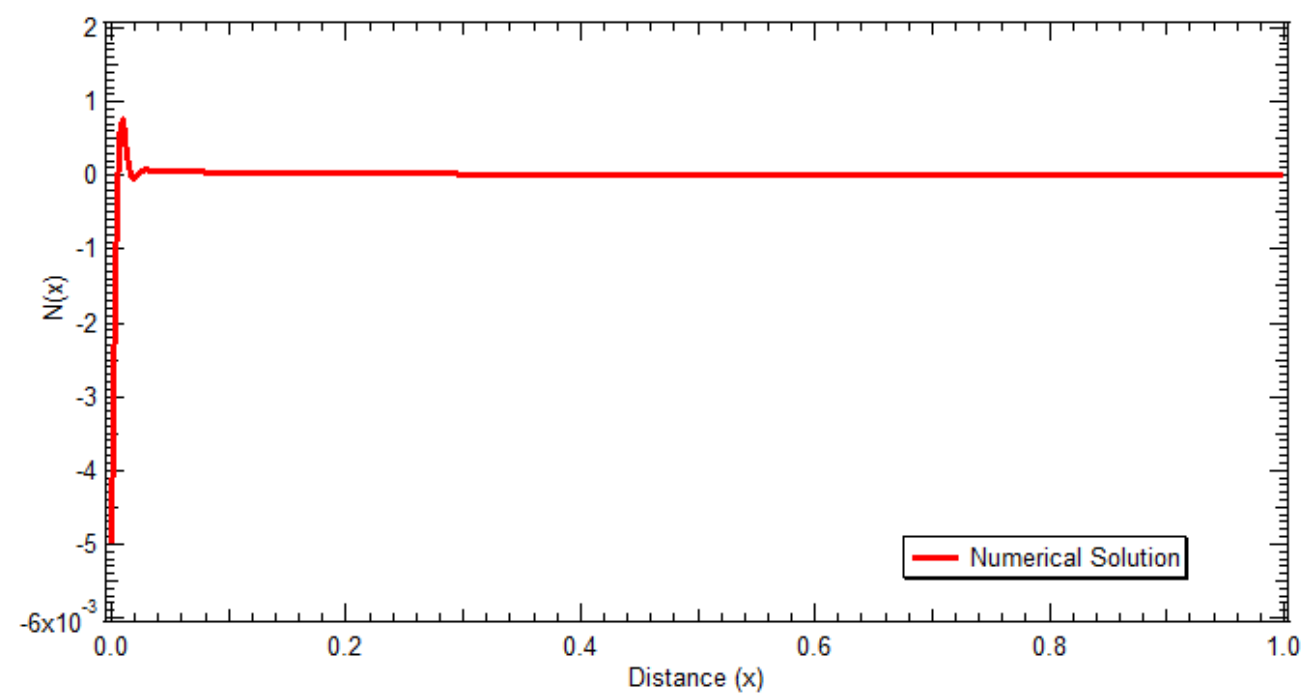

Figure 1. The numerical solution for $\mathrm{N}(\mathrm{x})$ resulting from solving the full PNP system (Equations 16-18). This solution can be compared to the analytical approximation of $\mathbf{N} \approx \mathbf{0}$

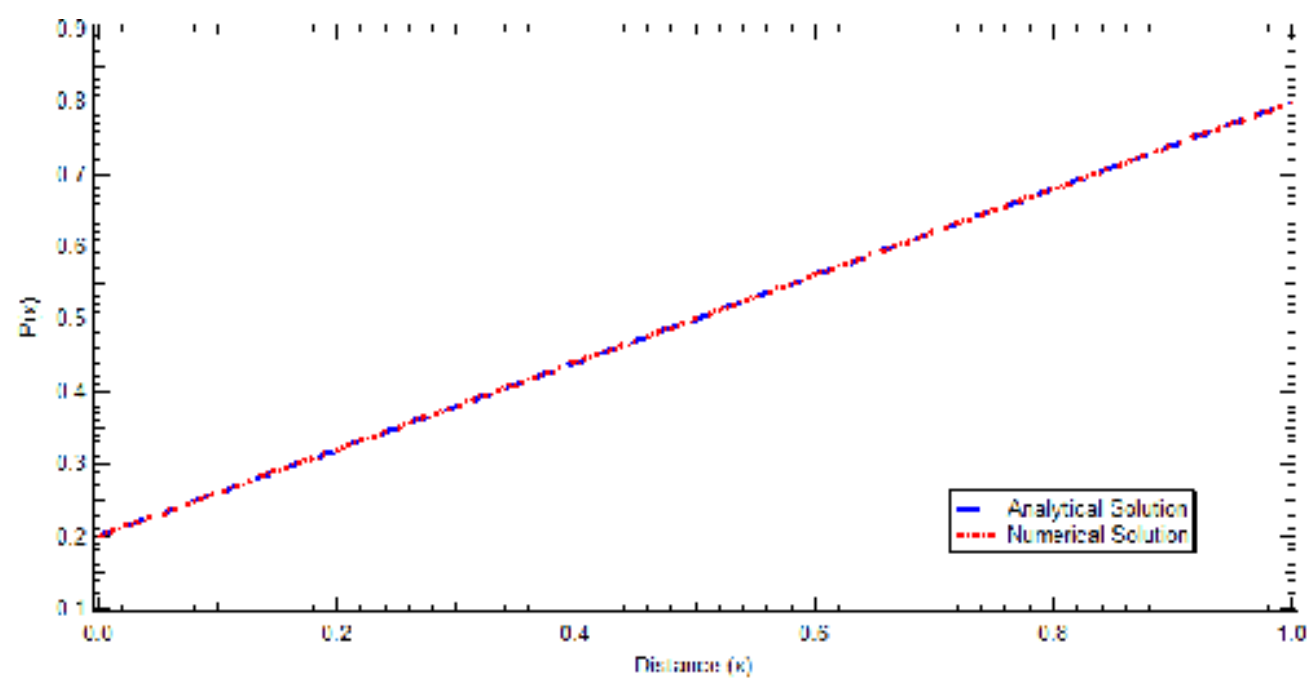

Figure 2. The numerical solution for N(x) resulting from solving the full PNP system (Equations 16-18) and the analytical solution for $\mathrm{P}(\mathrm{x})$ plotted from equation 19 


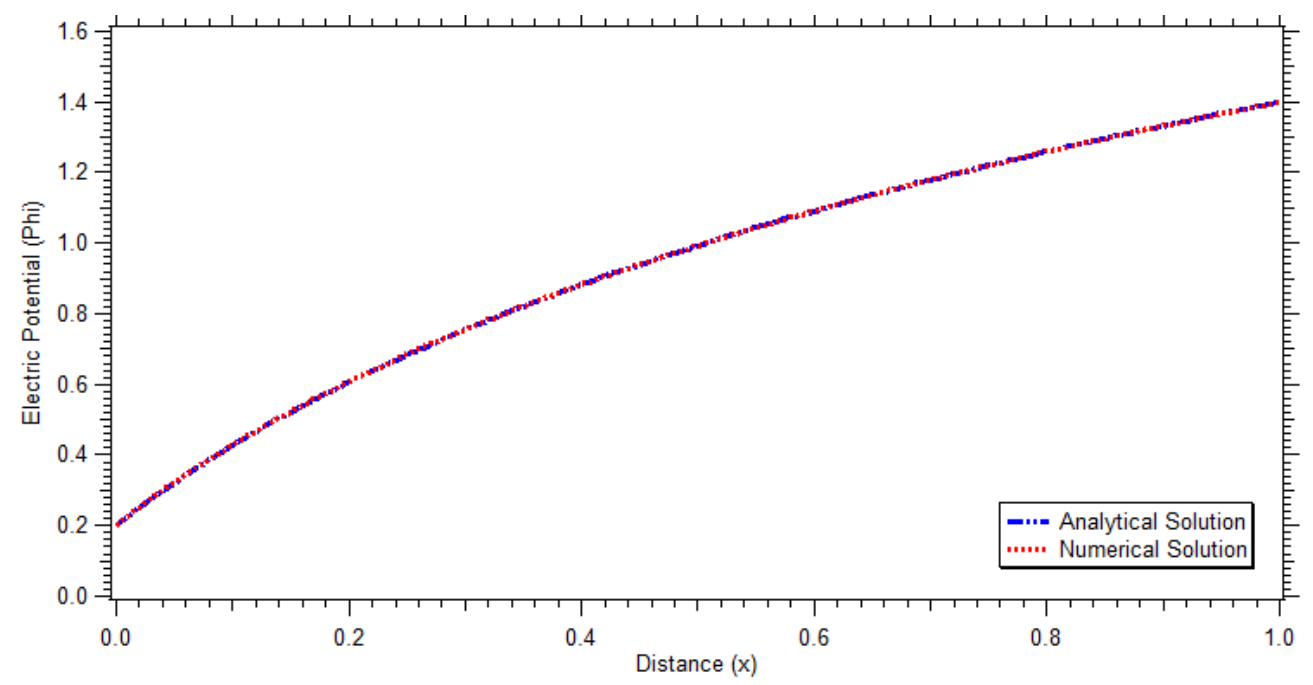

Figure 3. The numerical solution for the Electric Potential $\phi(\mathrm{x})$ resulting from solving the full PNP system

(Equations 16-18) and the analytical solution for the Electric Potential plotted from equation 20

\section{Poisson-Nernst-Planck Equations in Two Dimensions}

The PNP equations in two dimensions for two ionic species with $\mathrm{Z}=+1$ and $\mathrm{Z}=-1$ can be written as:

$$
\begin{gathered}
\frac{\partial p^{*}}{\partial t^{*}}=D_{p} \frac{\partial^{2} p^{*}}{\partial x^{* 2}}+D_{p} \frac{\partial^{2} p^{*}}{\partial y^{* 2}}+\frac{e D_{p}}{k T} \frac{\partial}{\partial x^{*}}\left(p^{*} \frac{\partial \varphi^{*}}{\partial x^{*}}\right)+\frac{e D_{p}}{k T} \frac{\partial}{\partial y^{*}}\left(p^{*} \frac{\partial \varphi^{*}}{\partial y^{*}}\right) \\
\frac{\partial n^{*}}{\partial t^{*}}=D_{n} \frac{\partial^{2} n^{*}}{\partial x^{* 2}}+D_{n} \frac{\partial^{2} n^{*}}{\partial y^{* 2}}+\frac{e D_{n}}{k T} \frac{\partial}{\partial x^{*}}\left(n^{*} \frac{\partial \varphi^{*}}{\partial x^{*}}\right)+\frac{e D_{n}}{k T} \frac{\partial}{\partial y^{*}}\left(n^{*} \frac{\partial \varphi^{*}}{\partial y^{*}}\right) \\
\frac{\partial^{2} \varphi^{*}}{\partial x^{* 2}}+\frac{\partial^{2} \varphi^{*}}{\partial y^{* 2}}=-\frac{e}{\varepsilon}\left(p^{*}-n^{*}\right)
\end{gathered}
$$

where the Einstein relations relating the ionic mobilities to the diffusivities have been used and the diffusivities and temperature are in turn taken to be constant. Using the same reference quantities for non-dimensionalization as described in Section 2.1.1 with the exception of $\mathrm{L}_{\mathrm{x}}$ and $\mathrm{L}_{\mathrm{y}}$ as the new characteristic length scales in the $\mathrm{x}$ and y directions, the PNP equations reduce to the following non-dimensional steady state form for the bioelectric dressing:

$$
\begin{gathered}
\frac{\partial^{2} p}{\partial x^{2}}+\Pi_{1} \frac{\partial^{2} p}{\partial y^{2}}+\Pi_{2} \frac{\partial}{\partial x}\left(p \frac{\partial \varphi}{\partial x}\right)+\Pi_{2} \Pi_{1} \frac{\partial}{\partial y}\left(p \frac{\partial \varphi}{\partial y}\right)=0 \\
\frac{\partial^{2} n}{\partial x^{2}}+\Pi_{1} \frac{\partial^{2} n}{\partial y^{2}}+\Pi_{2} \frac{\partial}{\partial x}\left(n \frac{\partial \varphi}{\partial x}\right)+\Pi_{2} \Pi_{1} \frac{\partial}{\partial y}\left(n \frac{\partial \varphi}{\partial y}\right)=0 \\
\frac{\partial^{2} \varphi}{\partial x^{2}}+\Pi_{1} \frac{\partial^{2} \varphi}{\partial y^{2}}=-\Pi_{5}(p-n)
\end{gathered}
$$

where $\Pi_{1}=\left(L_{x} / L_{y}\right)^{1 / 2}, \Pi_{2}=e \varphi_{r e f} / k T$, and $\Pi_{5}=e n_{r e f} L_{x}^{2} / \varepsilon \varphi_{r e f}$.

As in the case of the 1-D PNP equations, equations (24) and (25) may be added and subtracted to yield:

$$
\begin{gathered}
\frac{\partial^{2} P}{\partial x^{2}}+\Pi_{1} \frac{\partial^{2} P}{\partial y^{2}}+\Pi_{2} \frac{\partial}{\partial x}\left(N \frac{\partial \varphi}{\partial x}\right)+\Pi_{2} \Pi_{1} \frac{\partial}{\partial y}\left(N \frac{\partial \varphi}{\partial y}\right)=0 \\
\frac{\partial^{2} N}{\partial x^{2}}+\Pi_{1} \frac{\partial^{2} N}{\partial y^{2}}+\Pi_{2} \frac{\partial}{\partial x}\left(P \frac{\partial \varphi}{\partial x}\right)+\Pi_{2} \Pi_{1} \frac{\partial}{\partial y}\left(P \frac{\partial \varphi}{\partial y}\right)=0 \\
\frac{\partial^{2} \varphi}{\partial x^{2}}+\Pi_{1} \frac{\partial^{2} \varphi}{\partial y^{2}}=-\Pi_{5} N
\end{gathered}
$$

where $\mathrm{P}=\mathrm{p}+\mathrm{nN}=\mathrm{p}-\mathrm{n}$. These equations arise in the model problem described in the following section. 


\subsection{Model 2-D Problem}

\subsubsection{Formulation of Problem and Scaling}

The Procellera ${ }^{\circledR}$ bioelectric dressing consists of alternating circular regions of zinc oxide and silver oxide dots (on the order of millimeters in size and separated by millimeters) on a cloth base. When the dressing contacts wound exudate, redox reactions occur at the electrode surfaces that result in potential differences between silver and zinc dots (Banerjee et al., 2014). This results in an applied electric field distribution that drives electrotaxis and accelerates wound closure. For such a bioelectric dressing, $\phi_{\mathrm{ref}}=0.1 \mathrm{~V}, \mathrm{~T}=300 \mathrm{~K}, \mathrm{~L}_{\mathrm{x}}=1 \mathrm{~mm}, \mathrm{~L}_{\mathrm{y}}=100 \mu \mathrm{m}$, $\varepsilon \sim 80 \times 10^{-12} \mathrm{~F} / \mathrm{m}$, and $\mathrm{n}_{\text {ref }} \sim 10^{20} \mathrm{~m}^{-3}$.

Under these representative conditions:

$$
\Pi_{1}=\left(L_{x} / L_{y}\right)^{1 / 2} \sim O\left(10^{1}\right), \Pi_{2}=e \varphi_{\text {ref }} / k T \sim O(1), \text { and } \Pi_{5}=e n_{\text {ref }} L_{x}^{2} / \varepsilon \varphi_{\text {ref }} \sim O\left(10^{5}\right)
$$

From equation (29), these conditions correspond to the case of a quasi-neutral far-field where $\mathrm{N} \approx 0$ or $\mathrm{n} \approx \mathrm{p}$. Equations (27) - (29) then reduce to:

$$
\begin{gathered}
\frac{\partial^{2} P}{\partial x^{2}}+\epsilon^{2} \frac{\partial^{2} P}{\partial y^{2}}=0 \\
\frac{\partial}{\partial x}\left(P \frac{\partial \varphi}{\partial x}\right)+\epsilon^{2} \frac{\partial}{\partial y}\left(P \frac{\partial \varphi}{\partial y}\right)=0 \\
\frac{\partial^{2} \varphi}{\partial x^{2}}+\epsilon^{2} \frac{\partial^{2} \varphi}{\partial y^{2}}=0
\end{gathered}
$$

where $\epsilon=L_{y} / L_{x}=O\left(10^{-1}\right)$. The boundary conditions are taken to be $\varphi(x, y=0)=f(x)$, describing the potential distribution at the surface of the bioelectric dressing, $\varphi(x, y=1)=\varphi_{\infty}$, where $\varphi_{\infty}$ is the far-field potential which may be set to 0 for convenience. The remaining boundary conditions for are the symmetry

conditions: $\left.\frac{\partial \varphi}{\partial x}\right|_{x=0}=0$ and $\left.\frac{\partial \varphi}{\partial x}\right|_{x=1}=0$. The boundary conditions for $\mathrm{P}$ are taken to be $P(x, y=0)=g(x)$, where $\mathrm{g}(\mathrm{x})$ is a specified distribution of positive and negative ion concentrations at the surface of the bioelectric dressing. In the far-field, $\mathrm{P}$ is also taken to be specified, $\mathrm{P}(\mathrm{x}, \mathrm{y}=1)=2 \mathrm{n}_{\text {ref }}$. The remaining boundary conditions are

the symmetry conditions $\left.\frac{\partial P}{\partial x}\right|_{x=0}=0$ and $\left.\frac{\partial P}{\partial x}\right|_{x=1}=0$.

\subsubsection{Solution of 2-D Steady State PNP Equations}

The model problem described in Section 3.1.1 is solved using the method of singular perturbations (Mei, 1997). We first seek the outer solution in the far field, then the inner solution near the boundaries and finally find the uniformly valid solution through matching. The governing equation for the potential distribution is solved first. The outer solution can be found relatively easily by letting $\varphi(x, y)=\varphi_{0}(x, y)+\epsilon^{2} \varphi_{1}(x, y)+\ldots$

Substituting the perturbation expansion in equation (32) and applying the boundary conditions in the $\mathrm{x}$-direction, i.e. $\frac{\partial \varphi(x=0)}{\partial x}=\frac{\partial \varphi(x=1)}{\partial x}=0$, we arrive at:

$\mathrm{O}(1): \varphi_{0}(x, y)=C_{1}(y)$ (here $\mathrm{C}_{1}$ is a function of $(\mathrm{y})$ )

$\mathrm{O}\left(\varepsilon^{2}\right): \varphi_{1}(x, y)=-\frac{d^{2} C_{1}}{d y^{2}} \frac{x^{2}}{2}+C_{3}(y)$ with the solvability condition: $\frac{d^{2} C_{1}}{d y^{2}}=0$

Which yields the simple result: $C_{1}(y)=c_{1} y+c_{2}$

Hence we find that the zeroth order solution assumes the form:

$$
\varphi_{0}^{\text {OUT }}(x, y)=c_{1} y+c_{2}
$$

where $c_{1}$ and $c_{2}$ are constants independent of $\mathrm{y}$. Finally, applying the boundary condition $\varphi(x, y=1)=0$ away from the boundaries we arrive at the zeroth order outer solution:

$$
\varphi_{0}^{\text {OUT }}(x, y)=C(y-1)
$$

Next, we shift our attention to the boundary layer at $\mathrm{y}=0$. To examine this case, we introduce an inner variable: $Y=\frac{y}{\varepsilon}$ which leads to a Poisson equation of the form:

$$
\frac{\partial^{2} \varphi}{\partial x^{2}}+\frac{\partial^{2} \varphi}{\partial Y^{2}}=0
$$


Again, we assume the solution is a regular perturbation series in $\epsilon^{2}$ :

$$
\varphi(x, Y)=\varphi_{0}+\epsilon^{2} \varphi_{1}+\cdots
$$

We are interested in the leading term in the inner expansion. This leads to the following problem:

$$
\frac{\partial^{2} \varphi_{0}}{\partial x^{2}}+\frac{\partial^{2} \varphi_{0}}{\partial Y^{2}}=0
$$

subject to the following boundary conditions:

$$
\frac{\partial \varphi(x=0, Y)}{\partial x}=\frac{\partial \varphi(x=1, Y)}{\partial x}=0, \quad \varphi(x, Y=0)=f(x) \quad, \quad \varphi(x, Y \rightarrow \infty)=\text { finite }
$$

Equation (36) is solved using the technique of separation of variables to solve the Laplace equation for the leading term of the inner solution expansion which ultimately yields:

$$
\varphi(x, Y)=\frac{A_{0}}{2}+\sum_{n=1}^{\infty} A_{n} e^{-n \pi Y} \cos (n \pi x)
$$

where $A_{n}=2 \int_{0}^{1} f(x) \cos (n \pi x) d x$ for $\mathrm{n}=0,1,2, \ldots$ Finally, applying the matching condition:

$$
\lim _{Y \rightarrow \infty} \varphi_{0}(x, Y)=\lim _{y \rightarrow 0} \varphi_{0}^{O U T}(x, y)
$$

leads to a uniformly valid solution:

$$
\varphi(x, y)=\frac{A_{0}}{2}(1-y)+\sum_{n=1}^{\infty} A_{n} e^{-n \pi \frac{y}{\varepsilon}} \cos (n \pi x)+O\left(\varepsilon^{2}\right)
$$

The analytical solution given by equation (39) for the case of $f(x)=x$, and $\varphi_{0}=0$ is shown in Figure 4 . Figure 5 shows the numerical solution of the full Poisson-Nernst-Planck system (Equations 27-29) for the potential. As can be seen from the figures, the analytical solution shows good agreement with the numerical solution. To better quantify the agreement between the two solutions, the absolute value for the difference between the two is shown in Figure 6 . A maximum difference of about $3 \%$ is found near the $y=0$ boundary with much smaller differences everywhere else. Better agreement can be obtained by seeking the first order solution for the perturbation expansion, but in the spirit of keeping the results simple and useful for validation purposes, higher order terms in the expansion for the solution are not given here.

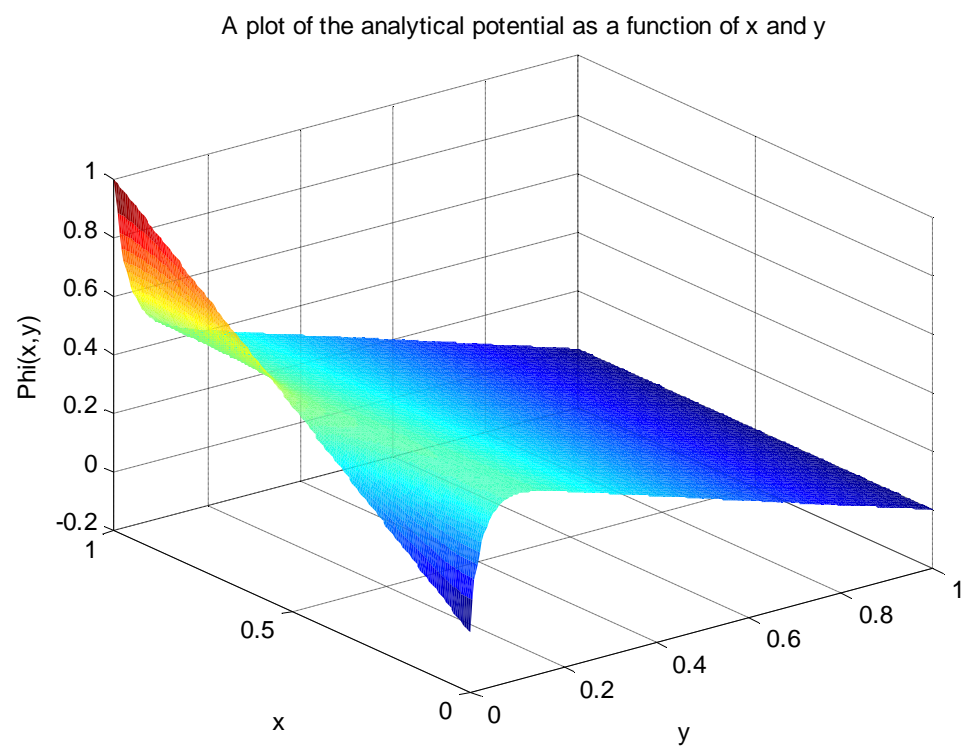

Figure 4. Analytical solution for the two dimensional PNP system of equations as obtained from equation (39) for the case of $\mathrm{f}(\mathrm{x})=\mathrm{x}$ and $\boldsymbol{\varphi}_{\infty}=\mathbf{0}$ 


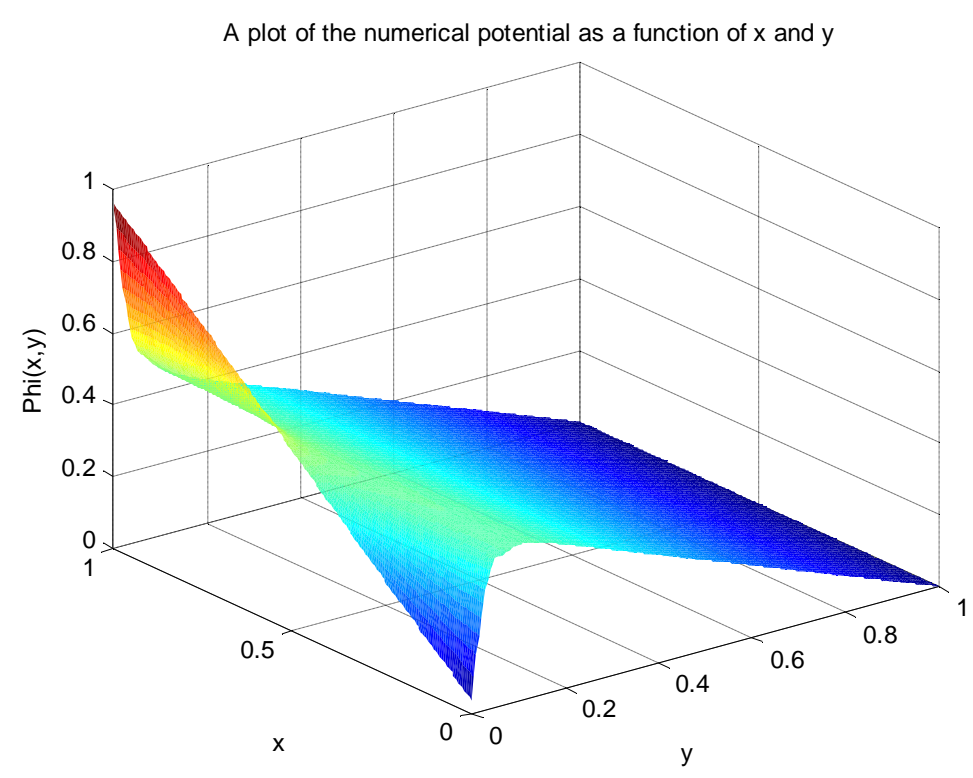

Figure 5. Numerical solution for the two dimensional PNP system of the system of equations (30-32) for the case of $\mathrm{f}(\mathrm{x})=\mathrm{x}$ and $\boldsymbol{\varphi}_{\infty}=\mathbf{0}$

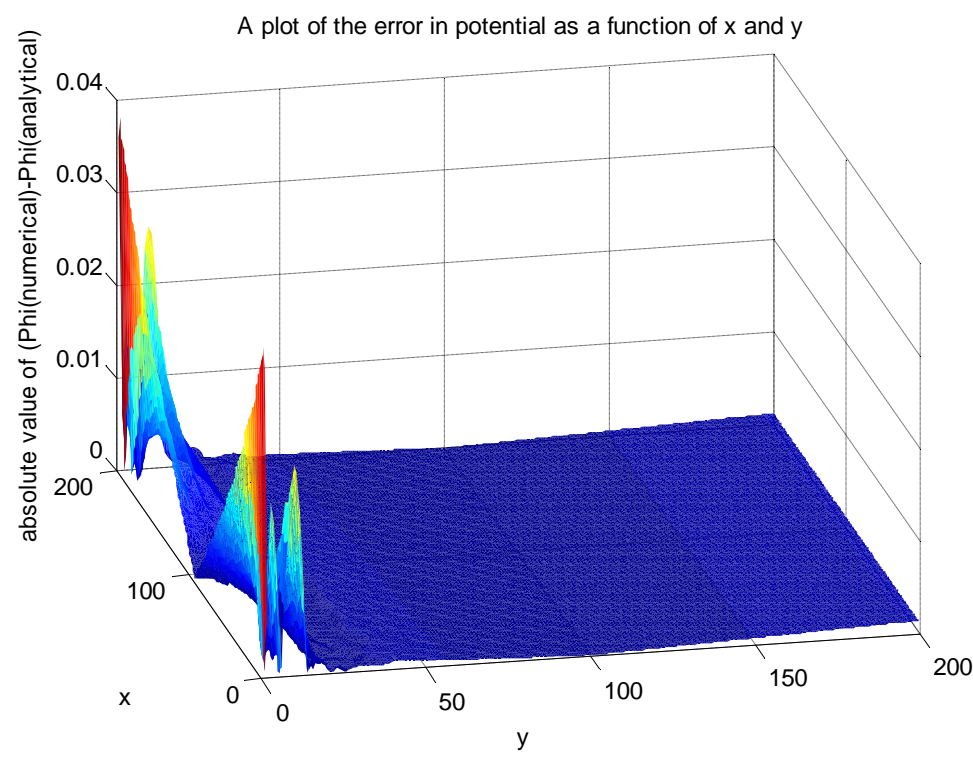

Figure 6. The absolute value for the difference between the numerical solution and the analytical solution shown in Figures 4 and 5

\section{Conclusions}

In this paper, analytical solutions for the steady state Poisson-Nernst-Planck equations in one and two dimensions have been given for model problems in the area of electrobiochemical systems such as bioelectric dressings used in would healing. These model problems can serve to benchmark and validate numerical solutions for more complex problems. The analytical solutions are obtained for two ionic species in both one and two dimensional systems which, to the best of our knowledge, have not been investigated in the literature. Simple closed form expressions are obtained in the one-dimensional case while an approximate solution using the method of singular perturbations is obtained for the two-dimensional model problem. The analytical solutions presented here are also shown to yield good agreement with numerical solutions of the corresponding PNP equations. The analytical solutions presented here may enable interpretation of experimental data for electrobiochemical systems. 


\section{Acknowledgements}

This work was supported in part by an IBM Faculty Award (V.S.) administered by Dr. T.C. Chen and Dr. T. J. Dalton.

\section{References}

Banerjee, J., Ghatak, P. D., Roy, S., Khanna, S., Sequin, E. K., Bellman, K., ... Sen, C. K. (2014). Improvement of Human Keratinocyte Migration by a Redox Active Bioelectric Dressing. PLoS ONE, 9(3), e89239. http://dx.doi.org/10.1371/journal.pone. 0089239

Barcilon, V., Chen, D. P., Eisenberg, R. S., \& Jerome, J. W. (1997). Qualitative properties of steady-state Poisson-Nernst-Planck systems: Perturbation and simulation study. SIAM Journal on Applied Mathematics, 57(3), 631-648.

Chein, R., \& Chung, B. (2013). Numerical study of ionic current rectification through non-uniformly charged micro/nanochannel systems. Journal of Applied Electrochemistry, 43(12), 1197-1206. http://dx.doi.org/10. 1007/s10800-013-0607-5

Coalson, R. D., \& Kurnikova, M. G. (2005). Poisson-Nernst-Planck theory approach to the calculation of current through biological ion channels. IEEE transactions on nanobioscience, 4(1), 81-93. http://dx.doi.org/10. 1109/TNB.2004.842495

Fíla, V., \& Bouzek, K. (2003). A mathematical model of multiple ion transport across an ion-selective membrane under current load conditions. Journal of Applied Electrochemistry, 33(8), 675-684. http://dx.doi.org/10.1023/a:1025018726112

Golovnev, A., \& Trimper, S. (2010). Steady state solution of the Poisson-Nernst-Planck equations. Physics Letters A, 374(28), 2886-2889. http://dx.doi.org/10.1016/j.physleta.2010.05.004

Golovnev, A., \& Trimper, S. (2011). Analytical solution of the Poisson-Nernst-Planck equations in the linear regime at an applied dc-voltage. The Journal of chemical physics, 134(15), 154902. http://dx.doi.org/10. $1063 / 1.3580288$

Hrdlička, J., Červenka, P., Přibyl, M., \& Šnita, D. (2010). Mathematical modeling of AC electroosmosis in microfluidic and nanofluidic chips using equilibrium and non-equilibrium approaches. Journal of Applied Electrochemistry, 40(5), 967-980. http://dx.doi.org/10.1007/s10800-009-9966-3

Krabbenhøft, K., \& Krabbenhøft, J. (2008). Application of the Poisson-Nernst-Planck equations to the migration test. Cement and Concrete Research, 38(1), 77-88. http://dx.doi.org/10.1016/j.cemconres.2007.08.006

Lee, S. H., \& Rasaiah, J. C. (2011). Proton transfer and the mobilities of the $\mathrm{H}+$ and $\mathrm{OH}-$ ions from studies of a dissociating model for water. The Journal of chemical physics, 135(12), 124505. http://dx.doi.org/10.1063/ 1.3632990

Liu, W. (2005). Geometric Singular Perturbation Approach to Steady-State Poisson-Nernst-Planck Systems. SIAM Journal on Applied Mathematics, 65(3), 754-766. http://dx.doi.org/10.1137/S0036139903420931

Markowich, P. A. (1986). The stationary semiconductor device equations. Wien; New York: Springer-Verlag.

Mei, C. C. ( 1997). Mathematical analysis in engineering : how to use the basic tools. Cambridge: Cambridge University Press.

Schönke, J. (2012). Unsteady analytical solutions to the Poisson-Nernst-Planck equations. Journal of Physics A: Mathematical and Theoretical, 45(45), 455204. http://dx.doi.org/10.1088/1751-8113/45/45/455204

Singer, A., Gillespie, D., Norbury, J., \& Eisenberg, R. S. (2008). Singular perturbation analysis of the steady-state Poisson-Nernst-Planck system: Applications to ion channels. European journal of applied mathematics, 19(05), 541-560. http://dx.doi.org/10.1017/S0956792508007596

Torabi, F., \& Aliakbar, A. (2012). A Single-Domain Formulation for Modeling and Simulation of Zinc-Silver Oxide Batteries. Journal of the Electrochemical Society, 159(12), A1986-A1992. http://dx.doi.org/10. $1149 / 2.038212 \mathrm{jes}$

Venkatraman, M., \& Van Zee, J. W. (2007). A model for the silver-zinc battery during high rates of discharge. Journal of Power Sources, 166(2), 537-548. http://dx.doi.org/10.1016/j.jpowsour.2006.12.064

\section{Copyrights}

Copyright for this article is retained by the author(s), with first publication rights granted to the journal.

This is an open-access article distributed under the terms and conditions of the Creative Commons Attribution license (http://creativecommons.org/licenses/by/3.0/). 